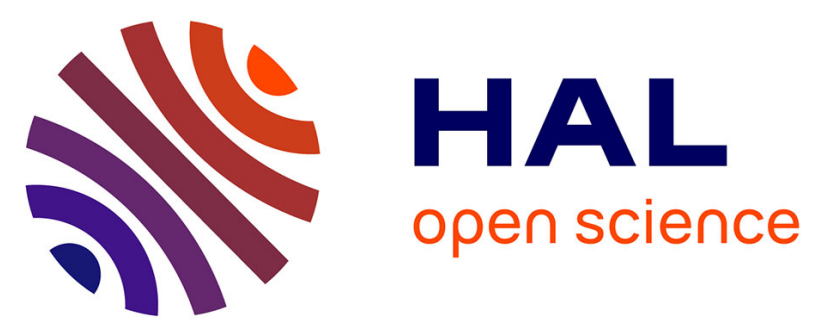

\title{
Spatial Extent and Temporal Correlation of Chorus and Hiss: Statistical Results From Multipoint THEMIS Observations
}

\author{
O. Agapitov, D. Mourenas, A. Artemyev, F. S. Mozer, J. W. Bonnell, V. \\ Angelopoulos, V. Shastun, V. Krasnoselskikh
}

\section{To cite this version:}

O. Agapitov, D. Mourenas, A. Artemyev, F. S. Mozer, J. W. Bonnell, et al.. Spatial Extent and Temporal Correlation of Chorus and Hiss: Statistical Results From Multipoint THEMIS Observations. Journal of Geophysical Research Space Physics, 2018, 123, pp.8317-8330. 10.1029/2018JA025725 . insu-03565611

\section{HAL Id: insu-03565611 https://hal-insu.archives-ouvertes.fr/insu-03565611}

Submitted on 11 Feb 2022

HAL is a multi-disciplinary open access archive for the deposit and dissemination of scientific research documents, whether they are published or not. The documents may come from teaching and research institutions in France or abroad, or from public or private research centers.
L'archive ouverte pluridisciplinaire HAL, est destinée au dépôt et à la diffusion de documents scientifiques de niveau recherche, publiés ou non, émanant des établissements d'enseignement et de recherche français ou étrangers, des laboratoires publics ou privés. 


\section{Journal of Geophysical Research: Space Physics}

\section{RESEARCH ARTICLE}

10.1029/2018JA025725

Key Points:

- Chorus and hiss amplitude statistics is presented based on 11 years of THEMIS VLF measurements

- Spatial extents of chorus and hiss waves have been determined in the statistical pattern

- Relation of chorus and hiss waves is shown in the statistical pattern

Correspondence to:

O. Agapitov,

oleksiy.agapitov@gmail.com

\section{Citation:}

Agapitov, O., Mourenas, D., Artemyev, A., Mozer, F. S., Bonnell, J. W., Angelopoulos, V., Shastun, V., \& Krasnoselskikh, V. (2018). Spatial extent and temporal correlation of chorus and hiss: Statistical results from multipoint THEMIS observations. Journal of Geophysical Research: Space Physics, 123, 8317-8330. https://doi.org/10.1029/ 2018JA025725

Received 28 MAY 2018 Accepted 25 SEP 2018

Accepted article online 3 OCT 2018

Published online 27 OCT 2018

\section{Spatial Extent and Temporal Correlation of Chorus and Hiss: Statistical Results From Multipoint THEMIS Observations}

\author{
O. Agapitov $^{1,2} \mathbb{D}$, D. Mourenas ${ }^{3}$ (D, A. Artemyev ${ }^{4}$ D, F. S. Mozer ${ }^{1}$ (D) J. W. Bonnell ${ }^{1}$, \\ V. Angelopoulos ${ }^{4}$, V. Shastun ${ }^{5}$ (D) and V. Krasnoselskikh ${ }^{5}$ (D) \\ ${ }^{1}$ Space Sciences Laboratory, University of California, Berkeley, CA, USA, ${ }^{2}$ Astronomy and Space Physics Department, \\ National Taras Shevchenko University of Kyiv, Kyiv, Ukraine, ${ }^{3}$ CEA, DAM, DIF, Arpajon, France, ${ }^{4}$ Institute of Geophysics and \\ Planetary Physics, University of California, Los Angeles, CA, USA, ${ }^{5}$ LPC2E/CNRS, Orleans, France
}

Abstract The spatial localization of whistler mode waves, determined by their generation process, propagation, and damping, is important for assessing the efficiency of wave-particle interactions and the dynamics of the radiation belts. We use 11 years of multipoint wave measurements in 2007-2017 from five Time History of Events and Macroscale Interactions during Substorms (THEMIS) spacecraft covering $L=2-10$ at low magnetic latitudes over all magnetic local times (MLTs) to characterize both the instantaneous spatial extent of chorus and hiss active regions and their wave amplitude distribution in the vicinity of their respective generation regions, making use of the time domain correlation technique. The correlation of lower band chorus wave power dynamics from two spacecraft measurements remains significant up to 250-800 km transverse to the background magnetic field, in agreement with the typical scale of a chorus element, with a shorter correlation length for higher peak wave power. Additionally, a coordinated analysis of average chorus amplitudes at separate locations shows that the radial extent of the chorus active region varies from $0.2 R_{\mathrm{E}}$ on the nightside to $1 R_{\mathrm{E}}$ on the dayside, with an azimuthal width of $1 \mathrm{MLT}$. The wave power correlation scale of hiss has no fine structure and is generally larger (1,500-3,000 km); its correlation remains nonnegligible up to $1.5 R_{\mathrm{E}}$. Such observations are consistent with chorus generation by localized electron injections and subsequent hiss amplification at lower $L$ shells from seed chorus waves having propagated and spread over a wider region inside the plasmasphere. The connection between hiss and chorus is further explored statistically through the correlation between their respective wave power variations, suggesting a significant link between them.

\section{Introduction}

The dynamics of particles population in the Van Allen radiation belts is controlled by losses (mostly due to precipitation of the particles into the upper atmosphere) and refilling due to external injections, transport, and local acceleration processes. Understanding the dynamics of electron fluxes is of particular importance, since these high-energy particles may pose a threat to satellites whose orbits cross the radiation belts. Favored mechanisms for driving in situ acceleration and pitch angle scattering loss are the interaction of electrons with naturally generated plasma waves in the whistler mode: chorus and hiss (e.g., Horne et al., 2005; Lyons \& Thorne, 1973; Mourenas et al., 2017). Chorus waves are observed as highly structured wave bursts with frequency rising or falling in time (called rising and falling tones, respectively) usually in two frequency bands separated by a wave power gap around $0.5 f_{\text {ce, }}$ corresponding to lower band chorus $(0.1-0.45$ of the equatorial $\left.f_{\mathrm{ce}}\right)$ and upper band chorus (0.5-0.9 of the equatorial $\left.f_{\mathrm{ce}}\right)$. They propagate from the geomagnetic equator roughly along the magnetic field (Agapitov et al., 2013; Burtis \& Helliwell, 1969; Helliwell, 1965; LeDocq et al., 1998; Santolík et al., 2005). Chorus emissions are observed in the inner magnetosphere outside the plasmapause throughout the dayside magnetosphere (Aryan et al., 2016; Meredith et al., 2001; Tsurutani \& Smith, 1974). Intensification of chorus generation in the inner and middle magnetosphere is associated with substorm electron injections (Goldstein \& Tsurutani, 1984; Meredith et al., 2001) and supposed to be carried out via the cyclotron instability caused by the temperature anisotropy of 10- to 50-keV electrons (e.g., Fu et al., 2014; Li et al., 2010; Sazhin \& Hayakawa, 1992). Generation of oblique chorus waves has also been observed in relation with field-aligned beams (or plateau distributions) of $\sim 1$-keV electrons and a temperature anisotropy of electrons below $10 \mathrm{keV}$ (Artemyev et al., 2016; Li et al., 2016; Mourenas et al., 2015). In order to quantify the parameters of wave-particle interactions with chorus, one must figure out and characterize 
their spatial extent. This spatial extent can also provide important additional information about the eventual presence of inhomogeneities, such as a possible fragmentation of hot electron injections during their propagation into the inner magnetosphere, their localization, or substructure (Gabrielse et al., 2014, 2017; Turner et al., 2017), and help to determine the most probable generation mechanisms responsible for the observed emissions.

This kind of information can be obtained making use of simultaneous very low frequency (VLF) measurements from multispacecraft missions through a correlation analysis. The first correlation study was performed based on a comparison of spectrograms from ISEE1 and ISEE2 for separation distances of about $400 \mathrm{~km}$. The comparison of chorus electric field spectrograms showed that on a timescale of a few minutes or longer, similar chorus emissions could be identified aboard both spacecraft, but on shorter timescales the detailed correlation tended to be poor, and the correlation scale was reported to be of the order of $100 \mathrm{~km}$ (Gurnett et al., 1979). This showed a need to distinguish the properties of a single chorus element (the spatial extent, amplitude distribution, and coherence extent) from the global scale of the chorus active region-the total area with similar wave amplitudes and frequency band. The scales of the generation region of a single chorus burst were estimated theoretically (Helliwell, 1965; Omura et al., 2013; Trakhtengerts, 1999) and experimentally based on the data from coordinated VLF measurements aboard Cluster (Agapitov et al., 2011; Artemyev et al., 2016; A. Breneman et al., 2007; Santolik et al., 2003, 2004; Santolík \& Gurnett, 2003), Time History of Events and Macroscale Interactions during Substorms (THEMIS; Agapitov et al., 2010), and the Van Allen Probes (Agapitov et al., 2017). Santolík and Gurnett (2003), Santolik et al. (2003, 2004), and Breneman et al. (2009) determined the correlation lengths of chorus-type whistler mode waves based on multipoint Cluster Wide-Band (WBD) Plasma Wave investigation in the vicinity of the chorus generation region during geomagnetic storms to be between approximately 60 and $120 \mathrm{~km}$, which was suggested to correspond to the size of the chorus generation region transverse to the background magnetic field (Santolik et al., 2003). Later, observations of similar chorus wave packets at separations larger than $500 \mathrm{~km}$ (Agapitov et al., 2011) indicated that the transverse size of the chorus source region is often larger in the outer radiation belt, where the typical source region scale was found to be around $600 \mathrm{~km}$ at $L \sim 4.5$ (Agapitov et al., 2011). Besides, Agapitov et al. (2010) used simultaneous observations of intense chorus by the four THEMIS spacecraft in the outer magnetosphere $(L>9)$. They estimated the transverse dimensions of chorus sources to be about 3,000 $\mathrm{km}$ there (wave frequency was about 0.15 of the equatorial $f_{\text {cer }}$ corresponding to a wavelength of about $300 \mathrm{~km}$ ). Recently, an analysis of long-lasting VLF waveforms ( $9 \mathrm{hr}$ of magnetic and electric field waveforms during 1 day) from the two Van Allen Probes have shown that a typical chorus burst can extend up to 600$800 \mathrm{~km}$ (for both lower and upper band chorus), while the amplitude of a given chorus element decays over a typical transverse scale of about $300 \mathrm{~km}$ (Agapitov et al., 2017). The statistical analysis of wave amplitude correlation based on continuous spectral data (one spectrum every $6 \mathrm{~s}$ calculated from 0.5 -s interval; Kletzing et al., 2013) recorded onboard the two Van Allen Probes during almost 3 years, and thus, related not to a single element but to the size of the global active region, found scales about $1,000-2,000 \mathrm{~km}$ for chorus in the outer radiation belt (Aryan et al., 2016). However, the spatial extent of the chorus wave active region has not yet been provided separately in both $L$ and magnetic local time (MLT).

Statistical information on the source size and active region extent is also lacking for hiss waves, which are intense broadband whistler mode waves observed in the region $L=2-5$ between about 100 and 2-3 kHz at frequencies smaller than $0.1 f_{\text {ce }}$ (Thorne et al., 1973; Meredith et al., 2004; Li et al., 2015). Hiss waves are thought to be amplified locally (Chen et al., 2012; Nakamura et al., 2016), probably from an embryonic source consisting of chorus waves having propagated inside the plasmasphere (Bortnik et al., 2008; Chen et al., 2012; Meredith et al., 2013). Hiss waves are mostly observed inside the plasmasphere but sometimes also at higher $L$ shells inside high-density plasmaspheric plumes within the 14-24 MLT sector (e.g., Moldwin et al., 2004; Su et al., 2018, and references therein). Hiss waves strongly contribute to electron losses inside the plasmasphere and the formation of a slot region between the inner and outer radiation belt, at least at energies below 12 MeV (Lyons \& Thorne, 1973; Ma et al., 2016; Mourenas et al., 2017). The present paper explores the fine structure of the chorus source scale, the distribution of the spatial scales of chorus and hiss, the extent of the wave active regions, and the connection between hiss and chorus in a statistical way, based on 11 years of THEMIS wave measurements over 2007-2017, using all five THEMIS spacecraft in 2008-2011 and three spacecraft since 2011. 

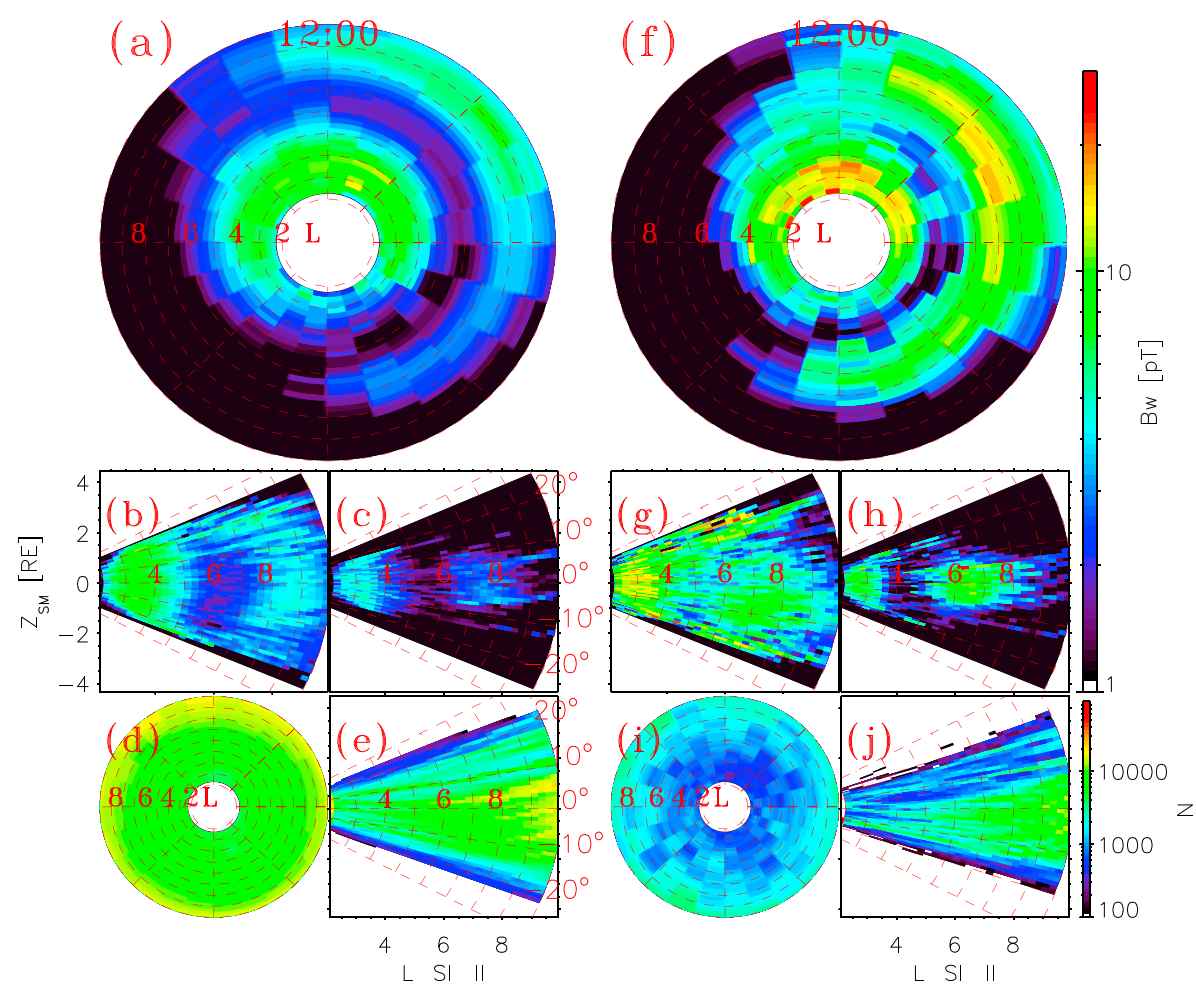

Figure 1. The distribution of lower-band chorus $\left(0.1 f_{\text {ce }}<f<0.5 f_{\text {ce }}\right)$ and hiss ( $\left.f_{\mathrm{LH}}<f<0.1 f_{\text {ce }}\right)$ amplitude in (a) MLT/L shell, and $\lambda / L$ shell domains for the (b) day 06-18 MLT and (c) night 18-06 MLT sectors of the magnetosphere during quiet $(K p<3)$ geomagnetic conditions. The coverage by the THEMIS VLF measurements in 2007-2017 in the (d) MLT/L shell and (e) $\lambda / L$ shell domains. (f-j) The distributions and coverage for perturbed $(K p>3)$ geomagnetic conditions in the same format as in panels (a)-(e). MLT = magnetic local time; THEMIS = Time History of Events and Macroscale Interactions during Substorms; VLF = very low frequency.

\section{Data Description}

The THEMIS mission began in February 2007 and consists of the five identically equipped National Aeronautics and Space Administration satellites (THA, THB, THC, THD, and THE). The main goal of this mission is to carry out multipoint investigations of substorm phenomena in the tail of the terrestrial magnetosphere (Sibeck \& Angelopoulos, 2008). For the current study, search coil magnetometer (SCM) observations (Roux et al., 2009) and electric field instrument measurements (Bonnell et al., 2008) in a bandwidth from 0.1 to $4 \mathrm{kHz}$ have been analyzed. Spin-averaged background magnetic field measurements with $4 \mathrm{~s}^{-1}$ sampling by the fluxgate magnetometers (FGMs; Auster et al., 2008) were used to determine the local electron cyclotron frequency $f_{\text {ce }}$ and lower hybrid frequency $f_{\mathrm{LH}}$. Continuous measurements of the magnetic and electric field mean wave power in six logarithmically spaced frequency ranges from $1 \mathrm{~Hz}$ to $4 \mathrm{kHz}$ of filter bank (FBK) data were used to evaluate chorus and hiss wave amplitudes. One-component measurements of magnetic and electric field in the spacecraft rotation plane were used. Such components are mostly directed transverse to the background magnetic field. This is due to the spacecraft orbit close to the equatorial plane and the spacecraft rotation axis transverse to the ecliptic plane. Whistler mode waves with a field-aligned propagation direction have an almost circular polarization of their magnetic field, with one component transverse to the background magnetic field direction. This allows to estimate whistler mode waves' magnetic field amplitudes from the FBK data (Li et al., 2009). In the present study, the FBK data obtained in 2007-2017 from all five probes are analyzed to obtain the parameters of the distribution of chorus waves over a radial range from 3 to $10 R_{\mathrm{E}}$ at magnetic latitudes $(\lambda)$ less than $20^{\circ}$. The magnetic latitude of the spacecraft is estimated based on a pure dipolar magnetic field model. Although this model may significantly underestimate the actual magnetic latitude in the presence of field-line stretching during strong geomagnetic disturbances, it is worth noting that there were almost no measured chorus waves at $L>7$ around local midnight (see Figures 1a and 1c). During 

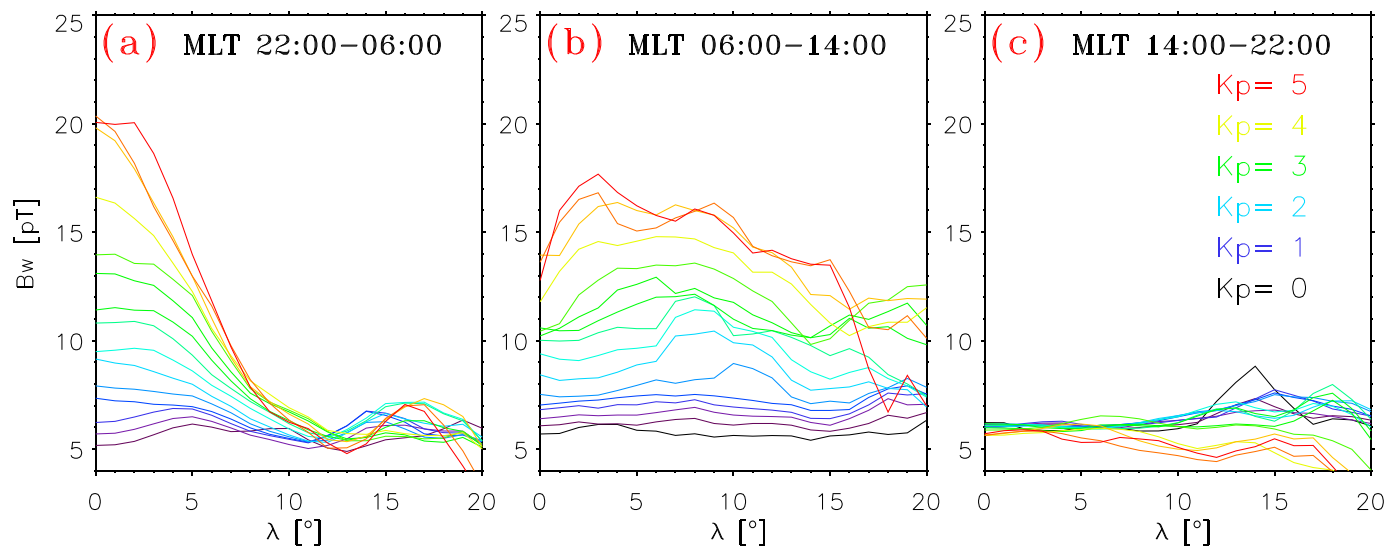

Figure 2. Distribution of chorus wave amplitude (at $L$ shell range from 5 to 10 ) in the (a) night-morning (2200-0600 MLT), (b) day (0600-1400 MLT), and (c) evening (1400-2200 MLT) sectors on geomagnetic latitude for the range of the geomagnetic activity level. MLT = magnetic local time.

these years, the gradually modified spacecraft orbits provided a comprehensive coverage of all MLTs. Simultaneous measurements were available from identical THEMIS spacecraft with an interspacecraft distance $|\Delta r|$ varying from tens of $R_{\mathrm{E}}$ down to about $100 \mathrm{~km}$. In the present study, chorus waves are categorized as waves with a frequency between $\sim 0.1$ and 0.5 times the equatorial $f_{\text {ce }}$ observed at $L>5$ outside the plasmasphere, whereas hiss waves must have a frequency strictly smaller than 0.1 times the equatorial $f_{\mathrm{ce}}$ (but larger than the lower hybrid frequency $f_{\mathrm{LH}}$ ) and be observed at $L<5$ inside the plasmasphere. The spacecraft was considered to be located inside the plasmasphere when the density derived from spacecraft potential measurements was above a threshold density $30 \mathrm{~cm}^{-3}$ (Li et al., 2009).

The distribution of chorus wave mean amplitude presented in Figure 1 agrees well with the distributions reported by Li et al. (2009), Li, Bortnik, et al. (2011), and Meredith et al. (2012) based on FBK as well as waveform (Li, Bortnik, et al., 2011) THEMIS measurements, showing a morning sector (00-13 MLT) maximum in agreement with that by Meredith et al. (2012), which was absent in the study by Li et al. (2009) simply due to a lack of THEMIS measurements in this sector during active time intervals in 2007-2008. The observed enhancement of wave amplitude with geomagnetic activity level from 5-7 to 20 pT (shown in Figure 2 for three MLT sectors where the distribution remains roughly similar when performing Kolmogorov-Smirnov comparisons; see Agapitov et al., 2015) is also in agreement with results based on measurements from Cluster satellites (Agapitov et al., 2013, 2015) and the Van Allen Probes (Agapitov et al., 2018; Aryan et al., 2014). The location of maximum chorus wave power shifts from $L \sim 8-10$ during quiet periods to $L \sim 6-8$ during high geomagnetic activity. The wave amplitude is localized in the vicinity of the geomagnetic equator at $|\lambda|<10^{\circ}$ in the night-morning MLT sector (especially during intermediate and high geomagnetic activity) and distributed over the whole latitudinal range from $-20^{\circ}$ to $+20^{\circ}$ covered by THEMIS measurements in the day sector. On the dayside (06-18 MLT), the chorus wave amplitude distribution has a local minimum near the equator (see Figures $2 \mathrm{~b}$ and $2 \mathrm{c}$ ) in agreement with results based on Cluster and Van Allen Probes measurements (Agapitov et al., 2013, 2015, 2017).

The distribution of hiss wave power in Figure 1 is also consistent with previous statistics from Meredith et al. (2013) mainly based on a much smaller 1.5-year THEMIS data set combined with 10 years of Cluster data. Hiss wave power is stronger in the day sector, with a gap of the order of $R_{\mathrm{E}}$ between its upper $L$ limit and lower $L$ limit of the chorus active region, as expected if inward-propagating chorus waves constitute an embryonic source for plasmaspheric hiss as ray-tracing simulations have suggested (Bortnik et al., 2008; Chen et al., 2012). One also finds that the region of strong hiss gets reduced from $L<5$ to $L<4$ during high geomagnetic activity as the plasmapause gets closer to the Earth, while the peak hiss power shifts from 10 to 12 MLT.

\section{Spatial Scales of the Generation Regions of Chorus and Hiss}

One important advantage of the THEMIS mission is the availability of simultaneous measurements from five identical spacecraft with an interspacecraft distance $|\Delta r|$ varying from tens of $R_{\mathrm{E}}$ down to less than 

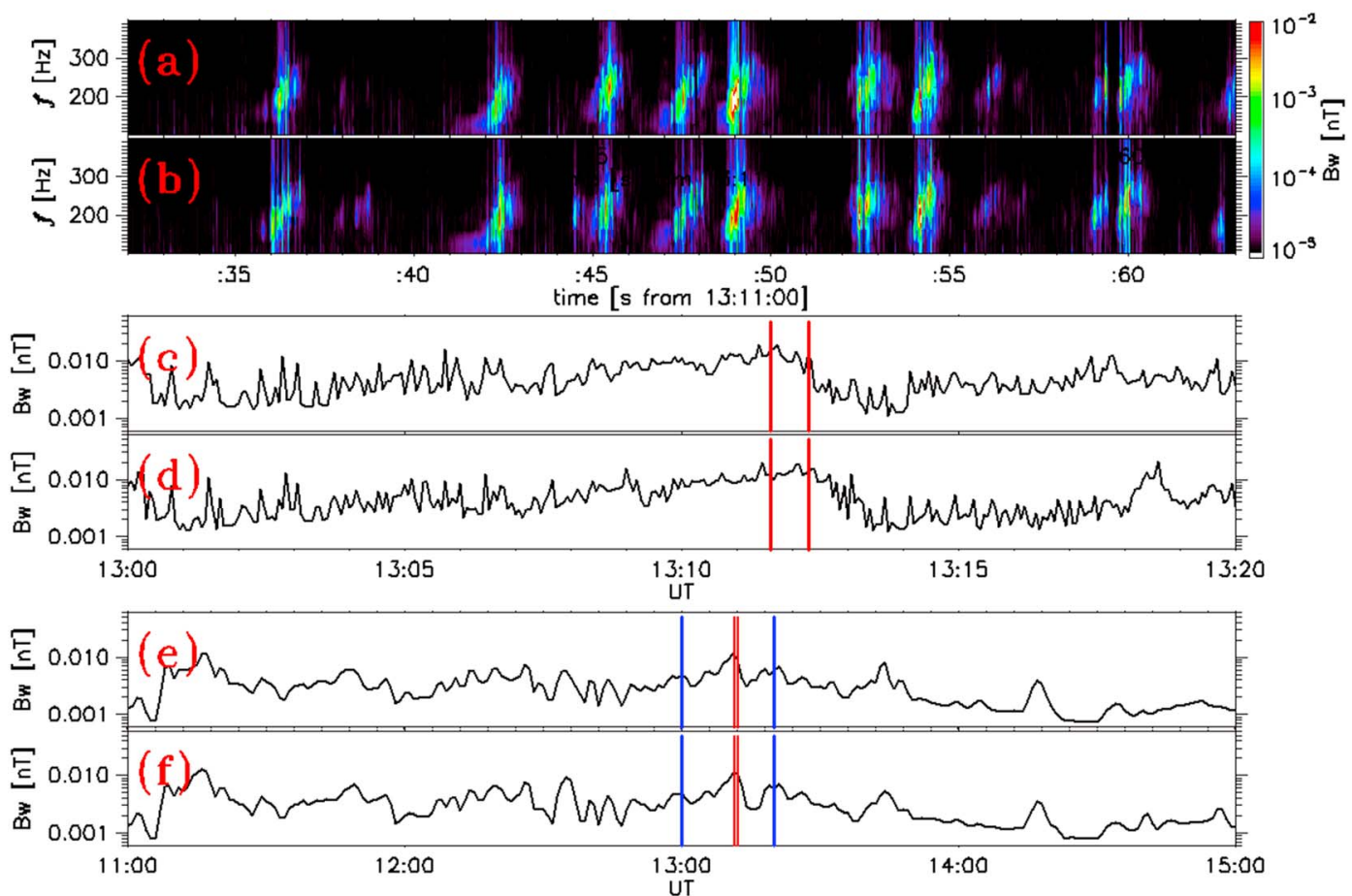

Figure 3. Dynamic spectra of magnetic field waveforms recorded aboard (a) THC and (b) THD on 17 June 2007. (c, d) The corresponding FBK wave power with $3 \mathrm{~s}^{-1}$ sampling. The time interval shown in (a) and (b) is marked by red lines in (c)-(f). $(e, f)$ The 1-min-averaged wave power over 11-15 UT, with the interval considered in $(c, d)$ marked by blue lines. FBK $=$ filter bank.

about $100 \mathrm{~km}$, mostly transverse to the background magnetic field in the inner magnetosphere. This gives the opportunity to study simultaneous and coordinated observations to determine the instantaneous distribution of wave parameters with no reliance on time-averaged data and, thus, to determine the instantaneous in situ radial profile of wave power as well as the typical scales of wave amplitude correlations.

Actually, chorus waves have a multiscale structure in time and space. The fine structure of the temporal as well as the spatial distribution of chorus is determined by the temporal and spatial extent of a single isolated chorus wave packet-a wave burst localized in time (0.1-0.3 s) with rising or falling frequency. Studies based on the measurements by Cluster (Agapitov et al., 2011; Santolik et al., 2003; Santolík et al., 2005), THEMIS (Agapitov et al., 2010), and the Van Allen Probes (Agapitov et al., 2017) have shown that the same chorus burst can be observed on different spacecraft as far as 1,000 km apart. One example of similarities in the waveforms from THA, THC, THD, and THE recorded on 17 July 2007 is presented in Figure 3. At that time, the THEMIS spacecraft were crossing a chorus active region (see details in Agapitov et al., 2010) with interspacecraft distances ranging from 100 to $2,000 \mathrm{~km}$. The SCM instrument and electric field instrument aboard the spacecraft provide several burst intervals with waveforms recorded over about $32 \mathrm{~s}$ during each orbit, resulting in a relatively poor coverage of the equatorial region by simultaneous and coordinated waveform measurements. However, one can take advantage of the usual stability of average chorus and hiss wave properties during tens of seconds in most observations to use the FBK data for a correlation study of wave properties. Accordingly, we used data stored in three frequency channels of FBK that cover a frequency range $80 \mathrm{~Hz}<f<4,000 \mathrm{~Hz}$. Since $f_{\text {ce }}$ drops from $13 \mathrm{kHz}$ at $L=4$ to $1,100 \mathrm{~Hz}$ at $L=9$, we use data only from channels in the frequency range $0.1 f_{\text {ce }}<f<0.5 f_{\text {ce }}$ (the frequency range of lower band chorus) and $f_{\mathrm{LH}}<f<0.1 f_{\text {ce }}$ (corresponding to hiss).

The correlation coefficient was calculated making use of 2-min intervals of FBK signal in the chorus and hiss frequency ranges captured aboard two of the THEMIS spacecraft (normalized over each 50-km interval of interspacecraft distance). The statistical distribution of this correlation coefficient is presented in Figures $4 \mathrm{~b}$ and $4 \mathrm{~d}$ for chorus and in Figure $4 \mathrm{f}$ for hiss. The mean values of the distribution and the $95 \%$ confidence 

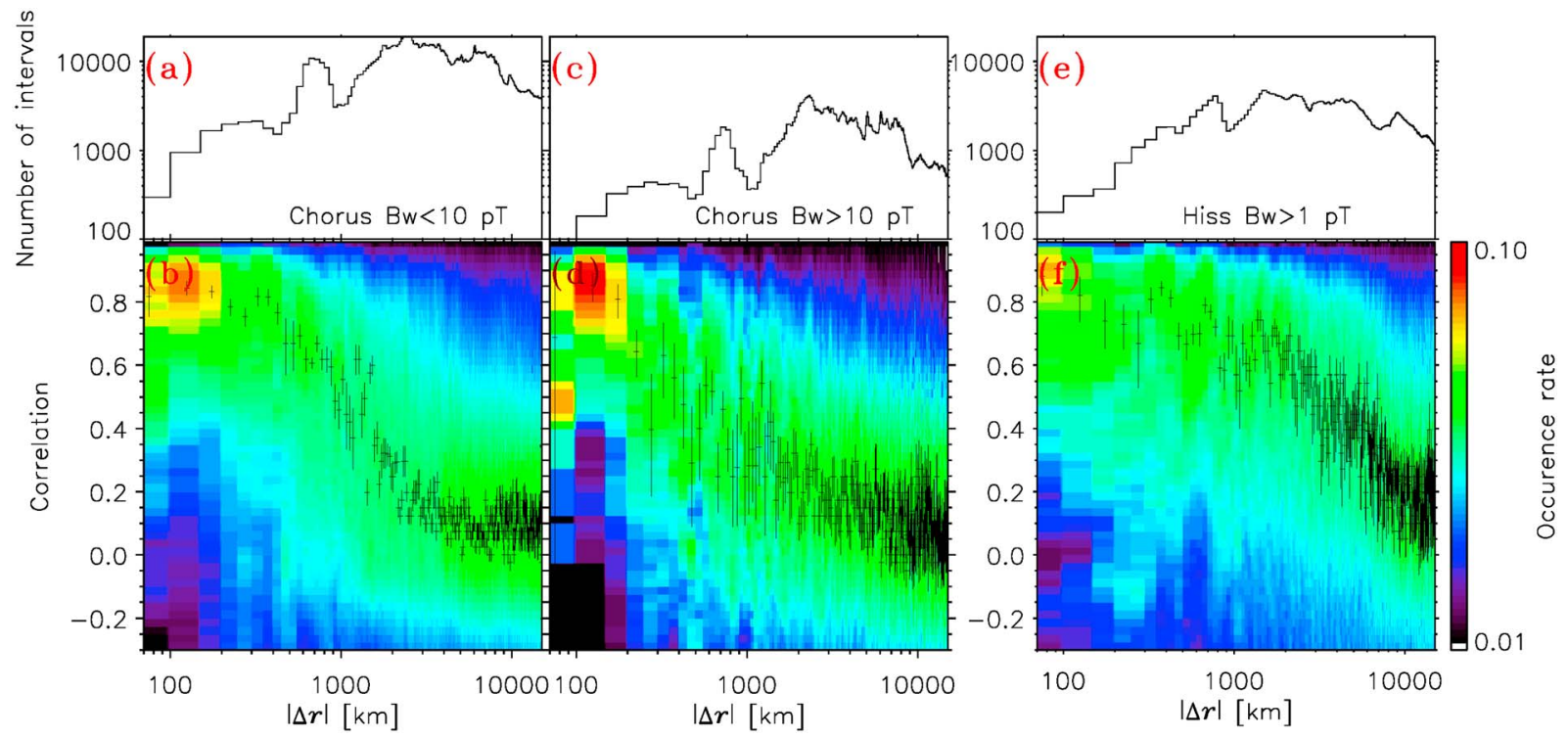

Figure 4. Total number of 2-min time series registered simultaneously by pairs of THEMIS probes separated by $|\Delta r|$ interspacecraft distance and stored in FBK during 2007-2017 for ( $a, c)$ lower-band chorus and (e) hiss. The distribution function of the cross-correlation coefficient of (b, d) chorus (for wave amplitudes $<10$ and $>10$ pT in panels (b) and (d), respectively) and ( $f$ ) hiss wave power signals captured on board each pair of THEMIS spacecraft as a function of interspacecraft distance $|\Delta r|$. The $95 \%$ confidence intervals of the mean values are shown by error bars, corresponding to twice the standard deviation $\sigma$ of the distribution. THEMIS $=$ Time History of Events and Macroscale Interactions during Substorms; FBK = filter bank.

interval are shown for each interval of interspacecraft distance. The corresponding numbers of processed intervals as a function of interspacecraft distance $|\Delta r|$ are presented in Figures $4 \mathrm{a}, 4 \mathrm{c}$, and $4 \mathrm{~d}$, respectively. The fast decay of the correlation as the $|\Delta r|$ increases up to $500-800 \mathrm{~km}$ agrees well with the characteristic transverse scale of individual chorus bursts reported by Agapitov et al. (2011, 2017). Measurements are available from identical THEMIS spacecraft for an interspacecraft distance $|\Delta r|$ varying from tens of $R_{\mathrm{E}}$ down to about $70 \mathrm{~km}$. Although the number of data points is maximal for $|\Delta r|>2,000-3,000 \mathrm{~km}$, it remains sufficient up to $|\Delta r|=2 R_{\mathrm{E}}$ and for $|\Delta r|>150 \mathrm{~km}$ at least for a reasonable statistical study (see Figures $4 a-4 c)$. Moreover, Figures $4 \mathrm{~b}$ and $4 \mathrm{~d}$ show that the radial scale of the correlation is significantly smaller $(\sim 250-400 \mathrm{~km})$ for chorus bursts with higher-amplitude ( $>10 \mathrm{pT})$ than for lower-amplitude bursts. One possible explanation is that chorus source regions of relatively moderate temperature anisotropy of injected hot $(5-30 \mathrm{keV})$ electrons (leading to weak linear wave growth) may naturally extend over larger distances than source regions of stronger temperature anisotropy (leading to fast wave growth). However, the smaller spatial extent of the source region producing chorus with larger wave amplitudes could also be related to the nonlinear stage of chorus wave generation and, in particular, the wave amplitude saturation process (e.g., see Omura et al., 2013; Trakhtengerts, 1999, and references therein). Besides, no significant variation of the scale of the chorus source region with $L$ shell or MLT has been found.

The scale of the mean correlation decay of hiss at $L$ shells $<5$ is much larger than for chorus in the radial direction, around 2,000-3,000 km in Figure 4f, with some significant correlation existing up to about $8,000 \mathrm{~km}$. Let us define here the size of the hiss active region as the maximum distance reached half of the time (i.e., for $50 \%$ of the observations) between simultaneous but distant observations of $>3$-pT hiss waves. Figures 5 and 6 show the instantaneous spatial extent of hiss and chorus in the radial and azimuthal directions, respectively. The comparison shows that they are rather similar, reaching about $1 R_{\mathrm{E}}$ in the radial direction and about $1.2 \mathrm{hr}$ of MLT in the azimuthal direction. Moreover, hiss waves are generally observed (at different universal times) over a twice larger MLT domain than are chorus waves-and mainly at slightly later MLTs, as expected for a generation of hiss from an embryonic source made of chorus waves mainly entering the plasmasphere at high latitudes around noon (Bortnik et al., 2008; Chen et al., 2012; Meredith et al., 2013).

The full distribution (in number of minutes of measurements) of the relative separation between two THEMIS spacecraft in azimuthal and radial directions is shown in Figures $7 \mathrm{a}$ and $7 \mathrm{~b}$, respectively, as a function of the $L$ 

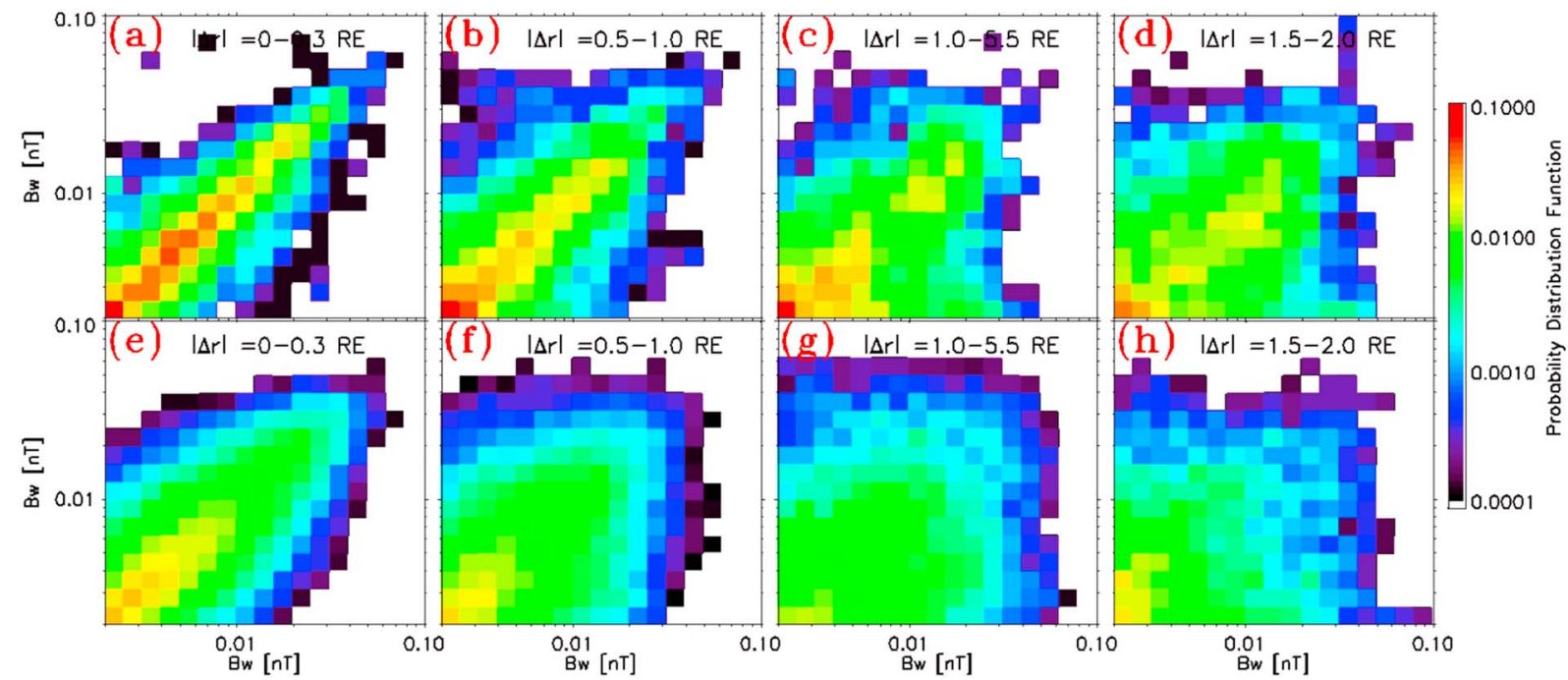

Figure 5. The cross-distributions of (a-d) hiss and (e-h) lower-band chorus wave amplitude captured simultaneously aboard each pair of two THEMIS spacecraft for different interspacecraft distance ranges. THEMIS = Time History of Events and Macroscale Interactions during Substorms.

shell of the first considered spacecraft in 2007-2017. The combined coverage of interspacecraft relative separations in the $\triangle M L T-\Delta L$ domain (with $\Delta L=L_{i}-L_{j}$ and $\Delta M L T=M L T_{i}-M T_{j}$, where $i$ and $j$ subscripts denote the two THEMIS spacecraft) is presented in Figures $7 \mathrm{c}$ and $7 \mathrm{~d}$ for chorus $(L>5$ and $22<M L T<14)$ and hiss $(L<5$ and MLT $>10)$ separately. The coverage of the measurements allows to provide significant results in the processed range of interspacecraft distances $|\Delta \mathrm{MLT}|<2$ and $|\Delta L|<2$.
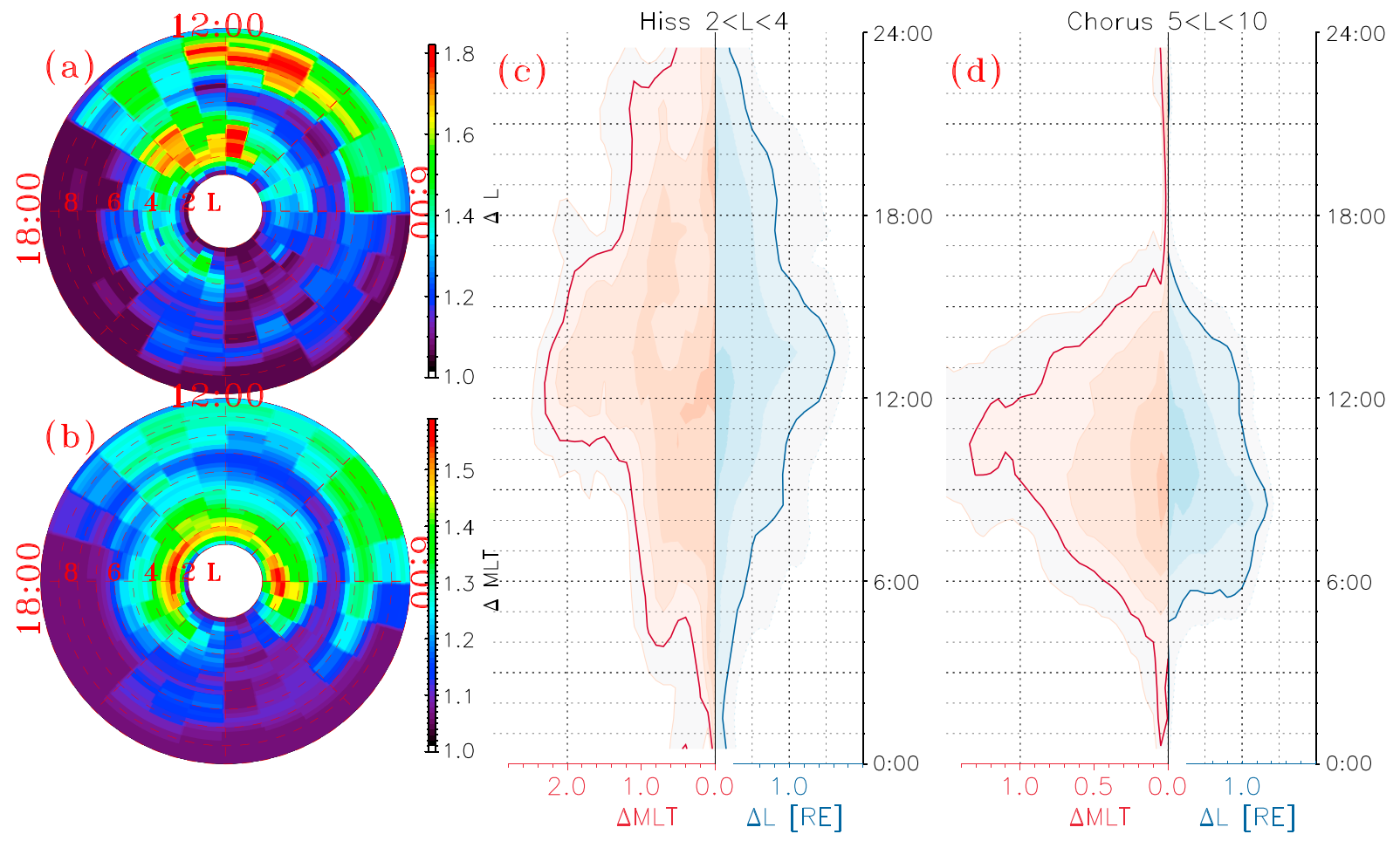

Figure 6. Spatial extent in the (a) radial and (b) azimuthal directions of the whistler mode waves, determined as the maximal distance at which the waves were observed simultaneously in at least $50 \%$ of the observations with more than 3-pT amplitudes. The spatial extents (the $\Delta M L T$ and $\Delta L$, at which the probability to observe the wave activity simultaneously aboard two spacecraft decays to 0.5$)$ for (c) hiss $\left(f_{\mathrm{LH}}<f<0.1 f_{\mathrm{ce}}\right)$ and (d) lower-band chorus $\left(0.1 f_{\mathrm{ce}}<f<0.5 f_{\mathrm{ce}}\right)$ active regions. $M L T=$ magnetic local time. 

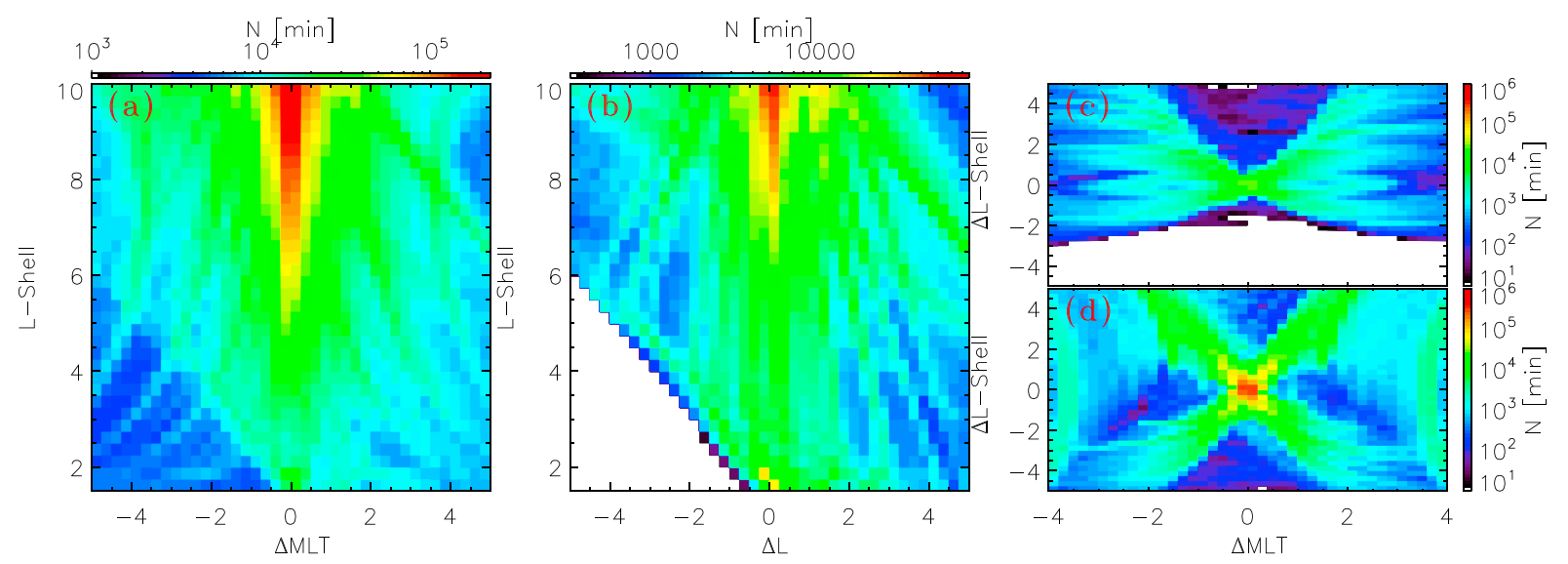

Figure 7. Distribution (in number $N$ of minutes) of relative separations between two THEMIS spacecraft in the (a) azimuthal ( $\triangle M L T)$ and (b) radial ( $\Delta L$ ) directions as a function of the $L$ shell of the first spacecraft (determined by alphabetical order, e.g., THEMIS C is before THEMIS D), for the available measurements in 2007-2017. The distribution of measurements in the $\Delta M L T-\Delta L$ domain for (c) chorus and (d) hiss. THEMIS = Time History of Events and Macroscale Interactions during Substorms.

\subsection{Correlations Between Chorus and Hiss Observations}

Next, we examine statistically the reliability of the model of hiss generation by local amplification from an embryonic source of chorus waves having propagated inside the plasmasphere (Bortnik et al., 2008, 2009; Chen et al., 2012 ; Meredith et al., 2013). Toward this goal, we provide in Figure 8a the probability over 2007-2017 to observe well-correlated chorus and hiss waves (i.e., with a correlation $>0.7$ between their wave power dynamics over $2 \mathrm{~min}$ ) onboard two separate THEMIS spacecraft, in the same whistler mode frequency band above $100 \mathrm{~Hz}$ corresponding to hiss waves ( $\mathrm{Li}$ et al., 2015), when one spacecraft was at $L=2-5$ inside the plasmasphere (corresponding to widespread hiss; see Figure 6) and the other spacecraft was at $L=6-9$ outside the plasmasphere (corresponding to widespread chorus; see Figure 6). We mostly consider chorus waves (with $0.1 f_{\text {ce }}<f<0.5 f_{\text {ce }}$ ) observed at $L>6$ and plasmaspheric hiss waves (with $f<0.1 f_{\text {ce }}$ ) observed at $L<5$. The plasmapause position was further checked using THEMIS spacecraft potential measurements, which provide an estimate of the electron density (Bonnell et al., 2008; Li et al., 2010; Li, Bortnik, et al., 2011). Since the proposed "seed" chorus waves must propagate inward and then get amplified inside the plasmasphere over 5-10 s to produce hiss (Chen et al., 2012; Meredith et al., 2013), we only considered as significant the chorus waves occurring within $10 \mathrm{~s}$ before hiss and the hiss waves occurring during the $10 \mathrm{~s}$ following chorus. Moreover, we only considered waves with amplitudes $>1$ pT.

We processed similar frequency chorus and hiss waves with amplitudes $>1$ pT observed simultaneously inside and outside the plasmasphere at an interspacecraft distance larger than $2 R_{\mathrm{E}}$ and $\triangle \mathrm{MLT}$ smaller than $3 \mathrm{hr}$. A little more than 1,000,000 of such 2-min time intervals have been detected during 2007-2017, making use of different pairs of THEMIS spacecraft. Among them, 71,000 time intervals were found with a correlation coefficient greater than 0.5 between chorus and hiss wave power dynamics. Therefore, the global average probability of finding such a high correlation between chorus and hiss waves, calculated as the number of intervals of high correlation divided by the total number of intervals of chorus and hiss observations, is $\sim 7 \%$ - but it reaches $\sim 20 \%$ over the wide $(L, M L T)$ region of maximal occurrence rates in Figure $8 a$. The percentage of chorus waves highly correlated with hiss waves observed slightly later (called chorus-hiss) and of hiss waves highly correlated with chorus waves detected just before (called hiss-chorus) are presented in Figure $8 \mathrm{a}$. About $20 \%$ of chorus waves at $L=6.5-8.5$ and $12-16$ MLT are well correlated with hiss waves observed immediately afterward inside the plasmasphere, whereas there is a slightly lower probability $(\sim 12 \%)$ for hiss waves at $L=3-5$ and $10-20$ MLT to be well correlated with chorus waves detected just before. The probability of detecting hiss when chorus is observed is larger than the probability of detecting chorus when hiss is observed, presumably due to the smaller spatial extent of the chorus active region than the hiss active region already discussed in section 3 (see Figures 5,6 ). Besides, the $L-M L T$ regions of significant probability of well-correlated chorus and hiss observations are in good agreement with the active regions of chorus and hiss found by Meredith et al. (2013) during active periods ( $A E>100 \mathrm{nT}$ ) at low latitudes $<30^{\circ}$ (see Figure 1b and Agapitov et al., 2013, 2018). 


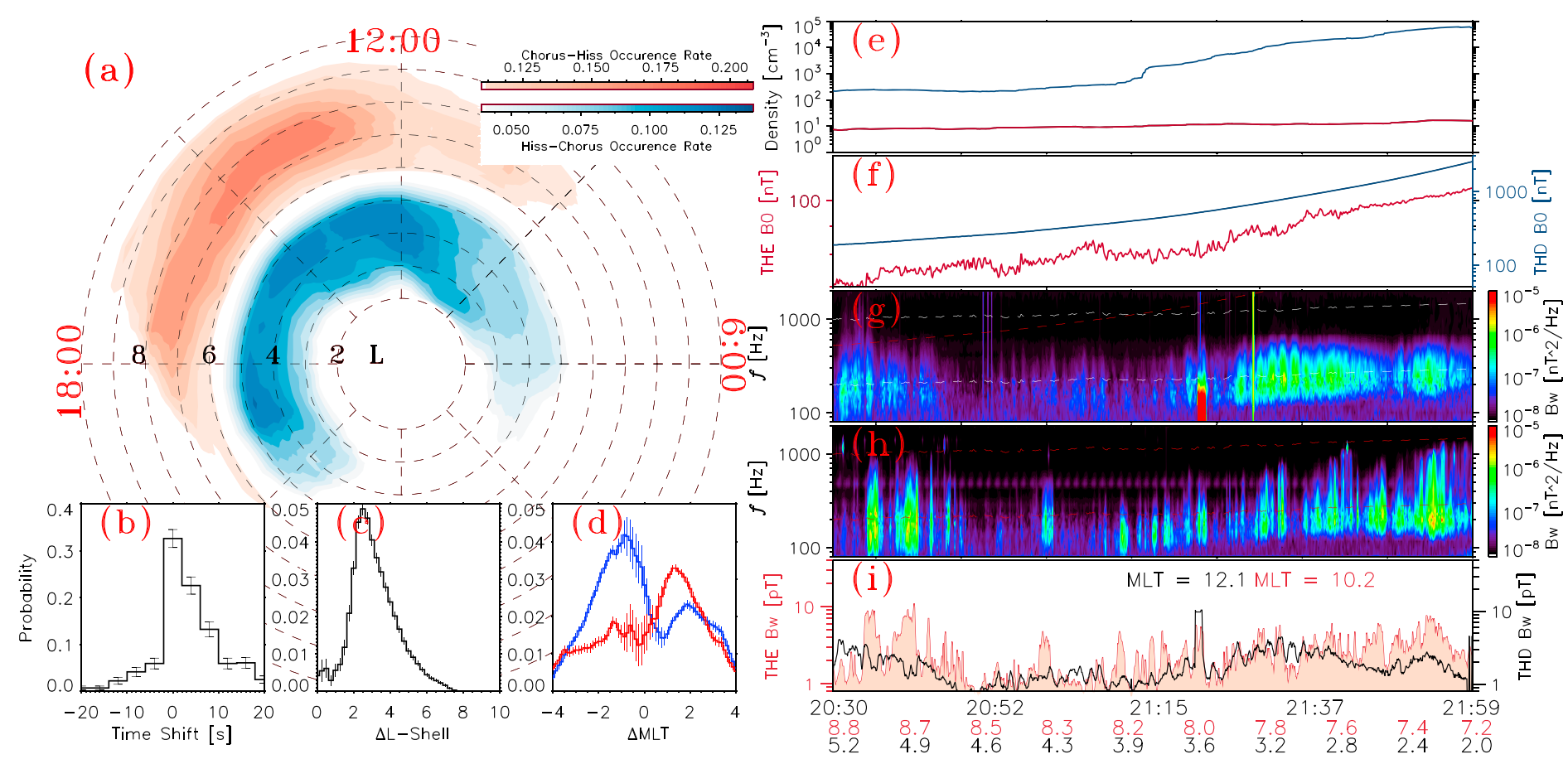

Figure 8. (a) Percentage of chorus waves (highlighted by red) such that their wave power dynamics is highly correlated with the wave power dynamics of hiss observed less than $10 \mathrm{~s}$ afterward inside the plasmasphere, and percentage of plasmaspheric hiss waves (highlighted by blue) similarly well correlated with chorus waves outside the plasmasphere (i.e., hiss occurring less than $10 \mathrm{~s}$ after chorus, with a high correlation $>0.7$ between their respective wave power dynamics). (b) The corresponding distribution of time delays between wave amplitude peaks of highly correlated chorus and plasmaspheric hiss. (c) The distribution of radial separation $\Delta L$ between well-correlated chorus and plasmaspheric hiss and (d) the corresponding distribution of azimuthal separation $\triangle M L T=M L T$ (chorus) - MLT (hiss), where the blue curve indicates measurements in the 00-12 MLT sector and the red curve shows measurements in the 12-24 MLT sector. An example of correlated chorus and plasmaspheric hiss observed during about an hour by THE (outside the plasmasphere; shown in red) and THD (inside the plasmasphere; marked in blue) is shown in panels (e)-(i). (e) The plasma density estimated from THD and THE spacecraft potential measurements. (f) The background magnetic field magnitude. The dynamics spectra of magnetic field perturbations recorded aboard $(\mathrm{g})$ THD and $(\mathrm{h}) \mathrm{THE}$. The red dashed curves indicate local values of $0.5 f_{\mathrm{ce}}$ and $0.05 f_{\text {ce }}$ in panel $(\mathrm{g})$ and the local $0.05 f_{\text {ce }}$ in panel $(\mathrm{h})$. The white dashed curves in panel $(\mathrm{g})$ show $0.5 f_{\text {ce }}$ and $0.05 f_{\text {ce }}$ values from panel $(\mathrm{h})$ corresponding to the background magnetic field at the location of THE. (i) The whistler mode wave power of chorus and hiss in the same frequency range of 100-1,000 $\mathrm{Hz}$.

In addition, Figure $8 \mathrm{~b}$ shows the distribution of time delay between each peak of chorus wave power and the temporally closest peak of hiss wave power recorded at lower $L$ shells during events of highly correlated chorus and hiss. This time delay is mostly between 0 and $6 \mathrm{~s}$, with a finite tail up to $20 \mathrm{~s}$. These results agree well with the time delay between chorus and hiss intensity peaks expected in the frame of a model of hiss generation through local amplification of seed chorus waves coming from outside the plasmasphere (Bortnik et al., 2008; Chen et al., 2012; Meredith et al., 2013). Figures 8c and 8d show that such well-correlated chorus and hiss waves are generally separated by $\sim 2-3 R_{\mathrm{E}}$ in the radial direction, hiss being observed mainly 1-2 hr later in MLT than chorus being in the 0-12 MLT sector but slightly more often at earlier MLT in the 12$24 \mathrm{MLT}$ sector. Hiss waves observed $\sim 1-2 \mathrm{hr}$ earlier in MLT than chorus in Figure $8 \mathrm{~d}$ can correspond to chorus active regions extending $\sim 1.0-1.5 \mathrm{hr}$ earlier in MLT than the location of chorus measurements, since Figure 6 has shown the existence of such large chorus extensions around noon. Therefore, these results mostly agree again with ray-tracing simulations of hiss generation through local amplification of seed chorus waves (Bortnik et al., 2008, 2011; Chen et al., 2012; Meredith et al., 2013).

One selected event-far from unique in our data set but with prolonged and very clear characteristic features-is displayed in Figures 8e-8i. Strong modulations (over $\sim 0.7$ - to 1.6-min time scales) of chorus wave power (in the frequency band $0.05 f_{\text {ce }}<f<0.5 f_{\text {ce }}$ ) are observed aboard the THE spacecraft at high $L=7-8$ outside the plasmasphere, well synchronized with modulations of hiss wave power (in the frequency band $\sim 100-500 \mathrm{~Hz}$ below the local $0.05 f_{\text {ce }}$ ) detected almost simultaneously at lower $L=2.4-$ 4.5 inside the plasmasphere aboard the THD spacecraft (the plasmapause was then around $L=5.5$ based on the density derived from spacecraft potential measurements and using a threshold density $\sim 30 \mathrm{~cm}^{-3}$ as in $\mathrm{Li}$ et al., 2009). The similar frequency band of the observed chorus and hiss emissions (compare 
Figures $8 \mathrm{~g}$ and $8 \mathrm{~h}$ ) actually corresponds to the local $0.05 f_{\text {ce }}-0.5 f_{\text {ce }}$ band estimated from background magnetic field measurements by THE outside the plasmasphere. The variation of the envelope of the wave power of plasmaspheric hiss over much longer time scales ( 15-50 min) turns out to be similarly well synchronized with the long-term variation of chorus wave power. The difference in $L$ shells between such highly correlated chorus and hiss varies from 3.5 to 4.5 , and hiss waves are observed $\sim 2 \mathrm{hr}$ later in MLT than chorus. All these global characteristics agree with the results of ray-tracing codes that show a possible generation of hiss through inward propagation of chorus waves (Bortnik et al., 2008; Chen et al., 2012; Meredith et al., 2013). Similarly, close relationships between chorus and hiss have also been found by analyzing the global response of these waves to interplanetary disturbances in recent event (Liu et al., 2017; Liu et al., 2017; Su et al., 2015) and statistical studies (Yue et al., 2017).

However, one important question remains: What is the cause of the strong wave power modulation of chorus and hiss waves in Figures 8g-8i? A similar well-correlated modulation of hiss waves has been observed recently on two Van Allen Probes located far away from each other, and it was suggested that then-observed ultralow frequency (ULF) waves in the Pc4-Pc5 band could have produced it through a modulation of plasma density that in turn modulates the number of resonant hot (10$100 \mathrm{keV}$ ) electrons and wave growth rates (Li et al., 2017). Weak density modulations were also seen at times by THE (not shown), simultaneously with ULF waves in the event of Figures 8e-8i. However, chorus growth rates can also be modulated by ULF waves through their modulation of the total magnetic field, which can slightly modify the cyclotron-resonant parallel electron velocity, eventually leading to modulations of the temperature anisotropy of the resonant hot electrons when this anisotropy varies with parallel electron velocity ( $\mathrm{Li}$ et al., 2011). During the event of Figures $8 \mathrm{e}-8 \mathrm{i}$, if ULF waves in the 10 to $25-\mathrm{mHz}$ range (e.g., Takahashi et al., 2015) had been simultaneously present over a broad $L$ range from $\sim 2.5$ to 9 , the similar wave power modulations of chorus and hiss could have been produced separately by similar ULF waves modulating their respective growth rates, without any cause-and-effect relationship between these two different waves. During the event in Figures $8 \mathrm{e}-8 \mathrm{i}$, however, strong ULF waves were measured aboard THE in the chorus source region at high $L$ shells, but no significant ULF waves were detected aboard THD inside the plasmasphere (see Figure 8f) where hiss wave power nevertheless exhibited a similar temporal modulation as chorus wave power at much higher $L$ (see Figure 8i). In the considered event, it is therefore likely that ULF waves present in the chorus source region first modulated chorus growth rates by varying the quantity and/or anisotropy of cyclotronresonant hot electrons (e.g., Li et al., 2017; Li, Thorne, et al., 2011; Xia et al., 2016), some of these power-modulated chorus waves later propagating inside the plasmasphere, where they provided a modulated seed of plasmaspheric hiss waves (Bortnik et al., 2008; Chen et al., 2012) accounting for the observed hiss modulation.

\section{Discussion and Conclusions}

In the present study, we examined the spatial parameters of chorus and hiss wave activity regions in a statistical way, making use of multispacecraft extremely low frequency/VLF measurements from the THEMIS project during 2007-2017. THEMIS extremely low frequency/VLF measurements in the FBK mode comprehensively covered all MLT and $L$ shells from 2 to 10 for a wide range of geomagnetic activity. The present distributions of chorus wave occurrence rate and amplitudes extend the databases provided by Li et al. (2009), Meredith et al. (2012), and Meredith et al. (2013) and show that chorus wave activity is regularly observed in the dawn-day sector of the magnetosphere consistent with earlier results (Li et al., 2009; Meredith et al., 2001, 2004; Andre et al., 2002; Green et al., 2005; Cully et al., 2008; Pokhotelov et al., 2008; Agapitov et al., 2011, 2012, 2013, 2015, 2018; Aryan et al., 2016). An enhancement of chorus activity with a shift of maximum wave amplitudes toward lower $L$ shells (from $L \sim 8-10$ to $L \sim 6-8$, partially following the plasmapause position) is observed during periods of higher geomagnetic activity. Lower-band chorus wave power on the nightside is localized near the equator below $10^{\circ}$ of latitude and on the dayside lower-band chorus wave power is distributed at all latitudes $\left(|\lambda|<20^{\circ}\right)$ covered by THEMIS measurements with a local minimum of the wave amplitude at the geomagnetic equator and a maximum shifted to $\lambda \sim 5-10^{\circ}$ at $L$ from 5 to 8 . Hiss is observed mainly at $L<5$ in the day sector of the plasmasphere (06-22 MLT) with the plasmapause position as its outer edge, and it is uniformly distributed over the latitude range covered by THEMIS measurements. 
The correlation scales of the whistler mode waves were estimated, making use of multispacecraft analysis of the THEMIS FBK spectral power data collected during 2007-2017. The correlation scale of chorus wave power dynamics in the outer radiation belt $(4<L<8)$ has been found to be around $500-800 \mathrm{~km}$ on average but only $250-400 \mathrm{~km}$ for the strongest bursts (see Figures $4 \mathrm{a}$ and $4 \mathrm{~b}$ ), providing an estimate of the distance over which the same chorus waves can be detected. Such results are in good agreement with previous studies of the average transverse dimension (Agapitov et al., 2011, 2017) and strong-burst (storm time) transverse dimension (Santolik et al., 2004) of the chorus source region, where the spatial extent of a single chorus element was estimated based on a finer chorus waveform correlation analysis. This inner scale of the chorus structure is important for nonlinear wave-particle interactions and probably determines the scale of bursty electron precipitations observed at ionospheric altitudes under the form of pulsating auroras (Nishimura et al., 2010, 2011) and microbursts (A. W. Breneman et al., 2017; Kersten et al., 2011; Mozer et al., 2018) depending on the energy of precipitating electrons. The significant level of correlation of the average chorus wave amplitude is observed at interspacecraft distances up to 2,000-3,000 km, consistent with the results of a correlation study based on Van Allen Probes VLF spectral measurements (Aryan et al., 2016). We estimated the instantaneous spatial extent of a chorus or hiss wave active region as a function of $L$ shell and MLT, by evaluating the maximum interspacecraft distance where waves were detected simultaneously with amplitudes above 3 pT in more than $50 \%$ of observations. This spatial extent of the chorus active region, together with the corresponding average wave intensity, determines the actual effectiveness of wave-particle interactions at any given time in multidimensional quasi-linear diffusion codes of the radiation belts. The chorus wave active region was found to be $\sim 1 R_{\mathrm{E}}$ in radial and $\sim 1 \mathrm{MLT}$ in the azimuthal direction. It suggests that the effects of chorus waves on electrons (both precipitation and acceleration) are probably much more inhomogeneous (i.e., localized in $L$ and MLT) than usually assumed in radiation belt codes, although the global effects (like electron lifetimes and acceleration rates) may end up to be correctly evaluated when considering the time- and MLT-averaged chorus wave intensity.

The chorus active regions are also much more localized in the radial direction on the nightside $\left(<0.3 R_{\mathrm{E}}\right)$ compared to the dayside ( 0.9-1.2 $\left.R_{\mathrm{E}}\right)$, with a roughly constant MLT scale ( 1 MLT) over the 6-13 MLT sector. Such scales presumably correspond to the spatial spread of hot (3-30 keV) electrons injected during substorms, which generate the observed chorus waves. Our results are therefore consistent with hot electron injections occurring in the form of initially narrow spatial bunches (of small MLT and radial spread) between local midnight and dawn, probably corresponding to incoming localized depolarizing flux bundles produced by bursty reconnections in the magnetotail (Gabrielse et al., 2014, 2017; Turner et al., 2017). At their arrival, such mainly localized successive injections cannot produce an MLT-continuous active region of chorus waves. However, once the injected electrons of different energies and azimuthal drift velocities have drifted toward sufficiently higher MLTs and have simultaneously spread to nearby $L$ shells (due to convection electric fields, radial diffusion, or drift shell splitting), they can probably constitute relatively wide homogeneous spatial regions of chorus generation, explaining the observations of much wider (in MLT and $L$ ) chorus active regions after 6 MLT. Dispersed hot electron injections inside geosynchronous orbit may also be more frequent after $3 \mathrm{MLT}$ than near local midnight, as suggested by statistical observations (Gabrielse et al., 2014). In the future, it would be interesting to examine the statistics of hot electron distributions in details to assess the reliability of such a global scenario.

Hiss, observed as a wideband signal often without clear structure, does show a slower decay of its crosscorrelation with distance than chorus, with a mean correlation scale of 2,000-3,000 km and some significant correlation up to about $8,000 \mathrm{~km}$. The (instantaneous) spatial extent of the hiss active region at $L<5$ was found to be larger or comparable to that of chorus, up to $3 \mathrm{hr}$ of MLT in the azimuthal direction (with similar amplitudes), with a radial scale of about $1 R_{\mathrm{E}}$-although hiss waves can be observed at different times over a much larger MLT domain spanning the whole dayside, as also shown by Breneman et al., (2015) and Li et al. (2017). The nonuniformity of hiss wave power in MLT at any given time should generally have no significant impact on electron precipitation, because the time scale of hiss-induced precipitation is usually longer than 1 day for 0.02- to 5-MeV electrons (e.g., Mourenas et al., 2017), therefore much longer than their azimuthal drift period.

Significant temporal correlations have also been found between distant observations of chorus (outside the plasmasphere) and hiss (inside the plasmasphere). We indeed found that $20 \%$ of the observed chorus waves are well correlated with hiss waves detected less than $10 \mathrm{~s}$ afterward, with a correlation $>0.7$ between their 
respective wave power dynamics. Such well-correlated chorus and hiss waves are generally separated by $\sim 2-$ $3 R_{\mathrm{E}}$ in the radial direction, hiss being usually observed 1-2 hr later in MLT than chorus. The observed time delay between chorus and hiss wave power peaks recorded at distant $L$ shells mostly varied between 0 and $6 \mathrm{~s}$, with a finite tail up to $20 \mathrm{~s}$. All these results are in good agreement with a model of hiss generation through local amplification of seed chorus waves coming from outside the plasmasphere (Bortnik et al., 2008; Chen et al., 2012; Meredith et al., 2013). Moreover, we have discussed one interesting event during which observed ULF waves likely modulated chorus wave power at high $L$ shells, whereas no ULF waves were seen at lower $L$ shells, where hiss waves nonetheless exhibited an intensity modulation well synchronized with the distant chorus intensity modulation. In the considered event, ULF waves present in the chorus source region likely modulated chorus wave growth rates by varying the quantity and/or anisotropy of cyclotron-resonant hot electrons, some of these power-modulated chorus waves later propagating inside the plasmasphere and providing a modulated seed of plasmaspheric hiss waves (Bortnik et al., 2008; Chen et al., 2012). Temporal variations of hot electron flux or temperature anisotropy at high $L$ shells could also provide additional chorus power modulations - such variations could occur from a feedback of the generated chorus waves during and after the generation process, since chorus-electron interactions usually reduce the initial anisotropy (e.g., Fu et al., 2014) and can lead to a fast loss of hot electrons into the atmosphere (e.g., Artemyev et al., 2016; Breneman et al., 2017; Mozer et al., 2018; Nishimura et al., 2010, 2011). All these interesting conjectures will be worth further checking in the future with accurate multipoint measurements of waves and particles.

\section{Acknowledgments}

The work of O. A. was supported by the NASA grant NNX16AF85G and THEMIS NASA contract NAS5-02099. The work of A. A. was supported by the NASA grant 80NSSC18K1112 and THEMIS NASA contracts NAS5-02099 and 80NSSC18K1112. V. K. and V. S. acknowledge financial support from the European Union's Horizon 2020 research and innovation program under the grant agreement 637302. The data used in this paper are available at the CDAWeb site http://http://cdaweb.sci. gsfc.nasa.gov. We acknowledge C. W. Carlson and J. P. McFadden for use of ESA data; A. Roux and O. LeContel for use of SCM data; and K. H. Glassmeier, U. Auster, and W. Baumjohann for the use of FGM data provided under the lead of the Technical University of Braunschweig and with financial support through the German Ministry for Economy and Technology and the German Center for Aviation and Space (DLR) under contract 50 OC 0302.

\section{References}

Agapitov, O., Artemyev, A., Krasnoselskikh, V., Khotyaintsev, Y. V., Mourenas, D., Breuillard, H., et al. (2013). Statistics of whistler mode waves in the outer radiation belt: Cluster STAFF-SA measurements. Journal of Geophysical Research: Space Physics, 118, 3407-3420. https://doi.org/ 10.1002/jgra.50312

Agapitov, O. V., Artemyev, A. V., Mourenas, D., Mozer, F. S., \& Krasnoselskikh, V. (2015). Empirical model of lower band chorus wave distribution in the outer radiation belt. Journal of Geophysical Research: Space Physics, 120, 10,425-10,442. https://doi.org/10.1002/ 2015JA021829

Agapitov, O., Blum, L. W., Mozer, F. S., Bonnell, J. W., \& Wygant, J. (2017). Chorus whistler wave source scales as determined from multipoint Van Allen Probe measurements. Geophysical Research Letters, 44, 2634-2642. https://doi.org/10.1002/2017GL072701

Agapitov, O., Krasnoselskikh, V., Dudok de Wit, T., Khotyaintsev, Y., Pickett, J. S., Santolík, O., \& Rolland, G. (2011). Multispacecraft observations of chorus emissions as a tool for the plasma density fluctuations' remote sensing. Journal of Geophysical Research, 116, A09222. https://doi. org/10.1029/2011JA016540

Agapitov, O., Krasnoselskikh, V., Khotyaintsev, Y. V., \& Rolland, G. (2012). Correction to "A statistical study of the propagation characteristics of whistler waves observed by Cluster." Geophysical Research Letters, 39, L24102. https://doi.org/10.1029/2012GL054320

Agapitov, O., Krasnoselskikh, V., Zaliznyak, Y., Angelopoulos, V., Contel, O. L., \& Rolland, G. (2010). Chorus source region localization in the Earth's outer magnetosphere using THEMIS measurements. Annales Geophysicae, 28(6), 1377-1386. https://doi.org/10.5194/angeo-28$1377-2010$

Agapitov, O. V., Mourenas, D., Artemyev, A. V., Mozer, F. S., Hospodarsky, G., Bonnell, J., \& Krasnoselskikh, V. (2018). Synthetic empirical chorus wave model from combined Van Allen Probes and Cluster statistics. Journal of Geophysical Research: Space Physics, 123, 297-314. https:// doi.org/10.1002/2017JA024843

André, R., Lefeuvre, F., Simonet, F., \& Inan, U. S. (2002). A first approach to model the low-frequency wave activity in the plasmasphere. Annales Geophysicae, 20, 981-996. https://doi.org/10.5194/angeo-20-981-2002

Artemyev, A., Agapitov, O., Mourenas, D., Krasnoselskikh, V., Shastun, V., \& Mozer, F. (2016). Oblique whistler-mode waves in the Earth's inner magnetosphere: Energy distribution, origins, and role in radiation belt dynamics. Space Science Reviews, 200(1-4), 261-355. https://doi. org/10.1007/s11214-016-0252-5

Aryan, H., Sibeck, D., Balikhin, M., Agapitov, O., \& Kletzing, C. (2016). Observation of chorus waves by the Van Allen Probes: Dependence on solar wind parameters and scale size. Journal of Geophysical Research: Space Physics, 121, 7608-7621. https://doi.org/10.1002/ 2016JA022775

Aryan, H., Yearby, K., Balikhin, M., Agapitov, O., Krasnoselskikh, V., \& Boynton, R. (2014). Statistical study of chorus wave distributions in the inner magnetosphere using Ae and solar wind parameters. Journal of Geophysical Research: Space Physics, 119, 6131-6144. https://doi.org/ 10.1002/2014JA019939

Auster, H. U., Glassmeier, K. H., Magnes, W., Aydogar, O., Baumjohann, W., Constantinescu, D., et al. (2008). The THEMIS fluxgate magnetometer. Space Science Reviews, 141(1-4), 235-264. https://doi.org/10.1007/s11214-008-9365-9

Bonnell, J. W., Mozer, F. S., Delory, G. T., Hull, A. J., Ergun, R. E., Cully, C. M., et al. (2008). The electric field instrument (EFI) for THEMIS. Space Science Reviews, 141(1-4), 303-341. https://doi.org/10.1007/s11214-008-9469-2

Bortnik, J., Chen, L., Li, W., Thorne, R. M., \& Horne, R. B. (2011). Modeling the evolution of chorus waves into plasmaspheric hiss. Journal of Geophysical Research, 116, A08221. https://doi.org/10.1029/2011JA016499

Bortnik, J., Li, W., Thorne, R. M., Angelopoulos, V., Cully, C., Bonnell, J., et al. (2009). An observation linking the origin of plasmaspheric hiss to discrete chorus emissions. Science, 324(5928), 775-778. https://doi.org/10.1126/science.1171273

Bortnik, J., Thorne, R. M., \& Meredith, N. P. (2008). The unexpected origin of plasmaspheric hiss from discrete chorus emissions. Nature, 452(7183), 62-66. https://doi.org/10.1038/nature06741

Breneman, A., Kletzing, C. A., Chum, J., Santolik, O., Gurnett, D., \& Pickett, J. (2007). Multispacecraft observations of chorus dispersion and source location. Journal of Geophysical Research, 112, A05221. https://doi.org/10.1029/2006JA012058

Breneman, A. W., Crew, A., Sample, J., Klumpar, D., Johnson, A., Agapitov, O., et al. (2017). Observations directly linking relativistic electron microbursts to whistler mode chorus: Van Allen Probes and FIREBIRD II. Geophysical Research Letters, 44, 11,265-11,272. https://doi.org/ 10.1002/2017GL075001 
Breneman, A. W., Halford, A., Millan, R., McCarthy, M., Fennell, J., Sample, J., et al. (2015). Global-scale coherence modulation of radiation-belt electron loss from plasmaspheric hiss. Nature, 523(7559), 193-195. https://doi.org/10.1038/nature14515

Breneman, A. W., Kletzing, C. A., Pickett, J., Chum, J., \& Santolik, O. (2009). Statistics of multispacecraft observations of chorus dispersion and source location. Journal of Geophysical Research, 114, A06202. https://doi.org/10.1029/2008JA013549

Burtis, W. J., \& Helliwell, R. A. (1969). Banded chorus-A new type of VLF radiation observed in the magnetosphere by OGO 1 and OGO 3. Journal of Geophysical Research, 74, 3002. https://doi.org/10.1029/JA074i011p03002

Chen, L., Li, W., Bortnik, J., \& Thorne, R. M. (2012). Amplification of whistler-mode hiss inside the plasmasphere. Geophysical Research Letters, 39, L08111. https://doi.org/10.1029/2012GL051488

Cully, C. M., Bonnell, J. W., \& Ergun, R. E. (2008). THEMIS observations of long-lived regions of large-amplitude whistler waves in the inner magnetosphere. Geophysical Research Letters, 35, L17S16. https://doi.org/10.1029/2008GL033643

Fu, X., Cowee, M. M., Friedel, R. H., Funsten, H. O., Gary, S. P., Hospodarsky, G. B., et al. (2014). Whistler anisotropy instabilities as the source of banded chorus: Van Allen Probes observations and particle in cell simulations. Journal of Geophysical Research: Space Physics, 119, 8288-8298. https://doi.org/10.1002/2014JA020364

Gabrielse, C., Angelopoulos, V., Harris, C., Artemyev, A., Kepko, L., \& Runov, A. (2017). Extensive electron transport and energization via multiple, localized dipolarizing flux bundles. Journal of Geophysical Research: Space Physics, 122, 5059-5076. https://doi.org/10.1002/ 2017JA023981

Gabrielse, C., Angelopoulos, V., Runov, A., \& Turner, D. L. (2014). Statistical characteristics of particle injections throughout the equatorial magnetotail. Journal of Geophysical Research: Space Physics, 119, 2512-2535. https://doi.org/10.1002/2013JA019638

Goldstein, B. E., \& Tsurutani, B. T. (1984). Wave normal directions of chorus near the equatorial source region. Journal of Geophysical Research, 89, 2789-2810. https://doi.org/10.1029/JA089iA05p02789

Green, J. L., Boardsen, S., Garcia, L., Taylor, W. W. L., Fung, S. F., \& Reinisch, B. W. (2005). On the origin of whistler mode radiation in the plasmasphere. Journal of Geophysical Research, 110, 3201. https://doi.org/10.1029/2004JA010495

Gurnett, D. A., Anderson, R. R., Scarf, F. L., Fredricks, R. W., \& Smith, E. J. (1979). Initial results from the ISEE-1 and -2 plasma wave investigation Space Science Reviews, 23(1), 103-122. https://doi.org/10.1007/BF00174114

Helliwell, R. A. (1965). Whistlers and related ionospheric phenomena. USA: Stanford University Press.

Horne, R. B., Thorne, R. M., Shprits, Y. Y., Meredith, N. P., Glauert, S. A., Smith, A. J., et al. (2005). Wave acceleration of electrons in the Van Allen radiation belts. Nature, 437(7056), 227-230. https://doi.org/10.1038/nature03939

Kersten, K., Cattell, C. A., Breneman, A., Goetz, K., Kellogg, P. J., Wygant, J. R., et al. (2011). Observation of relativistic electron microbursts in conjunction with intense radiation belt whistler-mode waves. Geophysical Research Letters, 38, L08107. https://doi.org/10.1029/ 2011GL046810

Kletzing, C. A., Kurth, W. S., Acuna, M., MacDowall, R. J., Torbert, R. B., Averkamp, T., et al. (2013). The electric and magnetic field instrument suite and integrated science (EMFISIS) on RBSP. Space Science Reviews, 179(1-4), 127-181. https://doi.org/10.1007/s11214-013-9993-6

LeDocq, M. J., Gurnett, D. A., \& Hospodarsky, G. B. (1998). Chorus source locations from VLF Poynting flux measurements with the polar spacecraft. Geophysical Research Letters, 25(21), 4063-4066. https://doi.org/10.1029/1998GL900071

Li, J., Bortnik, J., Li, W., Thorne, R. M., Ma, Q., Chu, X., et al. (2017). Coherently modulated whistler mode waves simultaneously observed over unexpectedly large spatial scales. Journal of Geophysical Research: Space Physics, 122, 1871-1882. https://doi.org/10.1002/ 2016JA023706

Li, W., Bortnik, J., Thorne, R. M., \& Angelopoulos, V. (2011). Global distribution of wave amplitudes and wave normal angles of chorus waves using THEMIS wave observations. Journal of Geophysical Research, 116, A12205. https://doi.org/10.1029/2011JA017035

Li, W., Chen, L., Bortnik, J., Thorne, R. M., Angelopoulos, V., Kletzing, C. A., et al. (2015). First evidence for chorus at a large geocentric distance as a source of plasmaspheric hiss: Coordinated THEMIS and Van Allen Probes observation. Geophysical Research Letters, 42, $241-248$. https://doi.org/10.1002/2014GL062832

Li, W., Ma, Q., Thorne, R. M., Bortnik, J., Kletzing, C. A., Kurth, W. S., et al. (2015). Statistical properties of plasmaspheric hiss derived from Van Allen Probes data and their effects on radiation belt electron dynamics. Journal of Geophysical Research: Space Physics, 120, 3393-3405. https://doi.org/10.1002/2015JA021048

Li, W., Mourenas, D., Artemyev, A. V., Bortnik, J., Thorne, R. M., Kletzing, C. A., et al. (2016). Unraveling the excitation mechanisms of highly oblique lower band chorus waves. Geophysical Research Letters, 43, 8867-8875. https://doi.org/10.1002/2016GL070386

Li, W., Thorne, R. M., Angelopoulos, V., Bortnik, J., Cully, C. M., Ni, B., et al. (2009). Global distribution of whistler-mode chorus waves observed on the THEMIS spacecraft. Geophysical Research Letters, 36, L09104. https://doi.org/10.1029/2009GL037595

Li, W., Thorne, R. M., Bortnik, J., Nishimura, Y., \& Angelopoulos, V. (2011). Modulation of whistler mode chorus waves: 1 . Role of compressional Pc4-5 pulsations. Journal of Geophysical Research, 116, A06205. https://doi.org/10.1029/2010JA016312

Li, W., Thorne, R. M., Bortnik, J., Nishimura, Y., Angelopoulos, V., Chen, L., et al. (2010). Global distributions of suprathermal electrons observed on THEMIS and potential mechanisms for access into the plasmasphere. Journal of Geophysical Research, 115, A00J10. https://doi.org/ 10.1029/2010JA015687

Liu, N., Su, Z., Gao, Z., Zheng, H., Wang, Y., Wang, S., et al. (2017). Simultaneous disappearances of plasmaspheric hiss, exohiss, and chorus waves triggered by a sudden decrease in solar wind dynamic pressure. Geophysical Research Letters, 44, 52-61. https://doi.org/10.1002/ 2016GL071987

Liu, N., Su, Z., Gao, Z., Reeves, G. D., Zheng, H., et al. (2017). Shock-induced disappearance and subsequent recovery of plasmaspheric hiss: Coordinated observations of RBSP, THEMIS, and POES satellites. Journal of Geophysical Research: Space Physics, 122, 10,421-10,435. https:// doi.org/10.1002/2017JA024470

Lyons, L. R., \& Thorne, R. M. (1973). Equilibrium structure of radiation belt electrons. Journal of Geophysical Research, 78(13), $2142-2149$. https://doi.org/10.1029/JA078i013p02142

Ma, Q., Li, W., Thorne, R. M., Bortnik, J., Reeves, G. D., Kletzing, C. A., et al. (2016). Characteristic energy range of electron scattering due to plasmaspheric hiss. Journal of Geophysical Research: Space Physics, 121, 11,737-11,749. https://doi.org/10.1002/2016JA023311

Meredith, N. P., Horne, R. B., \& Anderson, R. R. (2001). Substorm dependence of chorus amplitudes: Implications for the acceleration of electrons to relativistic energies. Journal of Geophysical Research, 106(A7), 13,165-13,178. https://doi.org/10.1029/2000JA900156

Meredith, N. P., Horne, R. B., Bortnik, J., Thorne, R. M., Chen, L., Li, W., \& Sicard-Piet, A. (2013). Global statistical evidence for chorus as the embryonic source of plasmaspheric hiss. Geophysical Research Letters, 40, 2891-2896. https://doi.org/10.1002/grl.50593

Meredith, N. P., Horne, R. B., Sicard-Piet, A., Boscher, D., Yearby, K. H., Li, W., \& Thorne, R. M. (2012). Global model of lower band and upper band chorus from multiple satellite observations. Journal of Geophysical Research, 117, A10225. https://doi.org/10.1029/2012JA017978

Meredith, N. P., Horne, R. B., Thorne, R. M., Summers, D., \& Anderson, R. R. (2004). Substorm dependence of plasmaspheric hiss. Journal of Geophysical Research, 109, A06209. https://doi.org/10.1029/2004JA010387 
Moldwin, M. B., Howard, J., Sanny, J., Bocchicchio, J. D., Rassoul, H. K., \& Anderson, R. R. (2004). Plasmaspheric plumes: CRRES observations of enhanced density beyond the plasmapause. Journal of Geophysical Research, 109, A05202. https://doi.org/10.1029/2003JA010320

Mourenas, D., Artemyev, A. V., Agapitov, O. V., Krasnoselskikh, V., \& Mozer, F. S. (2015). Very oblique whistler generation by low-energy electron streams. Journal of Geophysical Research: Space Physics, 120, 3665-3683. https://doi.org/10.1002/2015JA021135

Mourenas, D., Ma, Q., Artemyev, A. V., \& Li, W. (2017). Scaling laws for the inner structure of the radiation belts. Geophysical Research Letters, 44, 3009-3018. https://doi.org/10.1002/2017GL072987

Mozer, F. S., Agapitov, O. V., Blake, J. B., \& Vasko, I. Y. (2018). Simultaneous observations of lower band chorus emissions at the equator and microburst precipitating electrons in the ionosphere. Geophysical Research Letters, 45, 511-516. https://doi.org/10.1002/ 2017GL076120

Nakamura, S., Omura, Y., Summers, D., \& Kletzing, C. A. (2016). Observational evidence of the nonlinear wave growth theory of plasmaspheric hiss. Geophysical Research Letters, 43, 10,040-10,049. https://doi.org/10.1002/2016GL070333

Nishimura, Y., Bortnik, J., Li, W., Thorne, R. M., Chen, L., Lyons, L. R., et al. (2011). Multievent study of the correlation between pulsating aurora and whistler mode chorus emissions. Journal of Geophysical Research, 116, A11221. https://doi.org/10.1029/2011 JA016876

Nishimura, Y., Bortnik, J., Li, W., Thorne, R. M., Lyons, L. R., Angelopoulos, V., et al. (2010). Identifying the driver of pulsating aurora. Science, 330(6000), 81-84. https://doi.org/10.1126/science.1193186

Omura, Y., Nunn, D., \& Summers, D. (2013). Generation processes of whistler mode chorus emissions: Current status of nonlinear wave growth theory. In D. Summers, I. Mann, D. N. Baker, \& M. Schulz (Eds.), Dynamics of the Earth radiation belts and inner magnetosphere (pp. 243-254). Washington, DC: American Geophysical Union. https://doi.org/10.1029/2012GM001347

Pokhotelov, D., Lefeuvre, F., Horne, R. B., \& Cornilleau-Wehrlin, N. (2008). Survey of ELF-VLF plasma waves in outer radiation belt observed by Cluster STAFF-SA experiment. Annales Geophysicae, 26(11), 3269-3277. https://doi.org/10.5194/angeo-26-3269-2008

Roux, A., Le Contel, O., Coillot, C., Bouabdellah, A., de la Porte, B., Alison, D., et al. (2009). The search coil magnetometer for THEMIS. In The THEMIS mission, (pp. 265-275). New York, NY: Springer. DOI: https://doi.org/10.1007/978-0-387-89820-9_12

Santolík, O., \& Gurnett, D. A. (2003). Transverse dimensions of chorus in the source region. Geophysical Research Letters, 30(2), 1031. https:// doi.org/10.1029/2002GL016178

Santolik, O., Gurnett, D. A., \& Pickett, J. S. (2004). Multipoint investigation of the source region of storm-time chorus. Annales Geophysicae, 22, 2555-2563. Retrieved from https://hal-insu.archives-ouvertes.fr/docs/00/31/75/19/PDF/angeo-22-2555-2004.pdf

Santolik, O., Gurnett, D. A., Pickett, J. S., Parrot, M., \& Cornilleau-Wehrlin, N. (2003). Spatio-temporal structure of storm-time chorus. Journal of Geophysical Research, 108(A7), 1278. https://doi.org/10.1029/2002JA009791

Santolík, O., Gurnett, D. A., Pickett, J. S., Parrot, M., \& Cornilleau-Wehrlin, N. (2005). Central position of the source region of storm-time chorus Planetary and Space Science, 53(1-3), 299-305. https://doi.org/10.1016/j.pss.2004.09.056

Sazhin, S. S., \& Hayakawa, M. (1992). Magnetospheric chorus emissions: A review. Planetary and Space Science, 40(5), 681-697. https://doi.org/ 10.1016/0032-0633(92)90009-D

Sibeck, D. G., \& Angelopoulos, V. (2008). THEMIS science objectives and mission phases. Space Science Reviews, 141(1-4), 35-59. https://doi. org/10.1007/s11214-008-9393-5

Su, Z., Liu, N., Zheng, H., Wang, Y., \& Wang, S. (2018). Large amplitude extremely low frequency hiss waves in plasmaspheric plumes. Geophysical Research Letters, 45, 565-577. https://doi.org/10.1002/2017GL076754

Su, Z., Zhu, H., Xiao, F., Zheng, H., Wang, Y., Shen, C., et al. (2015). Disappearance of plasmaspheric hiss following interplanetary shock. Geophysical Research Letters, 42, 3129-3140. https://doi.org/10.1002/2015GL063906

Takahashi, K., Denton, R. E., Kurth, W., Kletzing, C., Wygant, J., Bonnell, J., et al. (2015). Externally driven plasmaspheric ULF waves observed by the Van Allen Probes. Journal of Geophysical Research: Space Physics, 120, 526-552. https://doi.org/10.1002/2014JA020373

Thorne, R. M., Smith, E. J., Burton, R. K., \& Holzer, R. E. (1973). Plasmaspheric Hiss. Journal of Geophysical Research, 78, 1581-1596. https://doi. org/10.1029/JA078i010p01581

Trakhtengerts, V. Y. (1999). A generation mechanism for chorus emission. Annales Geophysicae, 17(1), 95-100. https://doi.org/10.1007/ s00585-999-0095-4

Tsurutani, B. T., \& Smith, E. J. (1974). Postmidnight chorus: A substorm phenomenon. Journal of Geophysical Research, 79, 118-127. https://doi. org/10.1029/JA079i001p00118

Turner, D. L., Fennell, J. F., Blake, J. B., Claudepierre, S. G., Clemmons, J. H., Jaynes, A. N., et al. (2017). Multipoint observations of energetic particle injections and substorm activity during a conjunction between Magnetospheric Multiscale (MMS) and Van Allen Probes. Journal of Geophysical Research: Space Physics, 122, 11,481-11,504. https://doi.org/10.1002/2017JA024554

Xia, Z., Chen, L., Dai, L., Claudepierre, S. G., Chan, A. A., Soto-Chavez, A. R., \& Reeves, G. D. (2016). Modulation of chorus intensity by ULF waves deep in the inner magnetosphere. Geophysical Research Letters, 43, 9444-9452. https://doi.org/10.1002/2016GL070280

Yue, C., Chen, L., Bortnik, J., Ma, Q., Thorne, R. M., Angelopoulos, V., et al. (2017). The characteristic response of whistler mode waves to interplanetary shocks. Journal of Geophysical Research: Space Physics, 122, 10,047-10,057. https://doi.org/10.1002/2017JA024574 